\title{
Finance solidaire et système financier : une approche historique
}

Solidarity finance and the financial system : Insights from an historical approach

\section{Un enfoque histórico de la complementariedad entre finanza solidaria y sistema financiero}

\section{Amélie Artis}

Numéro 329, juillet 2013

Démocratie économique : un enjeu pour les entreprises... et pour les sciences sociales

The societal dimension of the social economy enterprise

URI : https://id.erudit.org/iderudit/1017934ar

DOI : https://doi.org/10.7202/1017934ar

Aller au sommaire du numéro

Éditeur(s)

Association Recma

ISSN

1626-1682 (imprimé)

2261-2599 (numérique)

Découvrir la revue

Citer cet article

Artis, A. (2013). Finance solidaire et système financier : une approche historique. Revue internationale de l'économie sociale, (329), 65-78. https://doi.org/10.7202/1017934ar
Résumé de l'article

La finance solidaire suscite un regain d'intérêt dans le contexte de crise actuelle, et elle est présentée comme une réponse innovante. Or ce constat est à nuancer. Depuis le XIX ${ }^{\mathrm{e}}$ siècle et aujourd'hui encore, en France, la finance solidaire développe une offre de financement complémentaire du fait des transformations de la régulation financière. Cette complémentarité par rapport au système financier conventionnel est-elle simplement un palliatif aux dysfonctionnements ou constitue-t-elle une réelle alternative dans les relations financières entre les emprunteurs et les intermédiaires financiers et dans l'organisation du système ? Par une approche historique comparée, nous montrons la permanence de la finance solidaire, tout en soulignant les formes de continuité et de discontinuité. Dès lors, nous discutons des rapports entre la finance solidaire et le système financier conventionnel, entre inclusion financière et transformations des règles. 


\section{FINANCE SOLIDAIRE ET SYSTĖME FINANCIER : UNE APPROCHE HISTORIQUE}

par Amélie Artis*

* Eseac, Pacte, Sciences-Po
Grenoble.Mél.:amelie.artis@
sciencespo-grenoble.fr

La finance solidaire suscite un regain d'intérêt dans le contexte de crise actuelle, et elle est présentée comme une réponse innovante. Or ce constat est à nuancer. Depuis le XIX siècle et aujourd'hui encore, en France, la finance solidaire développe une offre de financement complémentaire du fait des transformations de la régulation financière. Cette complémentarité par rapport au système financier conventionnel est-elle simplement un palliatif aux dysfonctionnements ou constitue-t-elle une réelle alternative dans les relations financières entre les emprunteurs et les intermédiaires financiers et dans l'organisation du système? Par une approche historique comparée, nous montrons la permanence de la finance solidaire, tout en soulignant les formes de continuité et de discontinuité. Dès lors, nous discutons des rapports entre la finance solidaire et le système financier conventionnel, entre inclusion financière et transformations des règles.

\section{Solidarity finance and the financial system : Insights from an historical approach}

The current recession has revived interest in solidarity finance as an innovative solution. However, this view needs to be qualified. Since the 19th century in France, solidarity finance has developed as a source of complementary finance as a result of changes in financial regulation. Is this complementary relationship with the conventional financial system simply a corrective for the system's shortcomings or a real alternative in the financial relationships between borrowers and financial intermediaries, and in the organisation of the system? Using a comparative historical approach, we show the permanency of solidarity finance while highlighting continuities and discontinuities. We then discuss the relationship between solidarity finance and the conventional financial system, which involves both financial inclusion and changes in the rules.

\section{Un enfoque histórico de la complementariedad entre}

finanza solidaria y sistema financiero

La finanza solidaria suscita un renovado interés en el contexto de la crisis actual, y es presentada como una respuesta innovadora. Ahora bien, este hallazgo debe ser matizado. Desde el siglo XIX hasta hoy, en Francia, la finanza solidaria desarrolla una oferta de financiamiento complementario, debido a las transformaciones de la regulación financiera. ¿Esta complementariedad respecto al sistema financiero convencional es simplemente un paliativo a los malos funcionamientos o ella constituye una real alternativa en las relaciones financieras entre los prestatarios y los intermediarios financieros y en la organización del sistema? Con un enfoque histórico comparativo, demostramos la permanencia de la finanza solidaria destacando las formas de continuidad y de discontinuidad. Por lo tanto, discutimos en este artículo, las relaciones entre la finanza solidaria y el sistema financiero convencional, entre inclusión financiera y transformaciones de las reglas. 
L'histoire récente montre une accélération du développement du système financier, avec la création de produits sophistiqués. Cette évolution a plutôt favorisé la volatilité et l'instabilité du système, au détriment du soutien à l'économie réelle. La crise financière de 2007 a eu deux impacts directs : une raréfaction de l'offre de crédit des banques et une dévalorisation brutale des actifs des emprunteurs (Aglietta, 2008). Dans ce contexte, des pratiques financières alternatives privées répondant aux besoins de financement des entrepreneurs ${ }^{(1)}$ sont réactivées et observée. Parmi elles, se dessine le champ de la finance solidaire.

Celle-ci se caractérise par un système de relations sociales de financement qui associe des relations monétaires et du lien social dans un ensemble cohérent (Artis, 2012). A la différence du financement classique, elle n'est pas une simple relation d'échange marchand anonyme. Elle s'inscrit dans un paradigme différent de la logique capitaliste, selon lequel la recherche de profit n'est pas la

(1) Dans cette contribution, nous ne prenons pas en considération la question des crédits à la consommation ou des crédits immobiliers, qui correspondent à des logiques différentes de notre objet. finalité de l'activité et les valeurs de réciprocité et de solidarité sont au centre des relations entre les prêteurs et les emprunteurs. Elle soutient des projets économiques viables qui subissent une contrainte de financement en raison de leurs spécificités (mode de gouvernance, propriété du capital, règles de redistribution des gains) par rapport à l'idéal-type de la société de capitaux. Aujourd'hui, la finance solidaire suit un sentier de croissance ininterrompue de ses activités avec une hausse de 32,9\% de l'épargne solidaire, une collecte d'environ 4,7 milliards d'euros début 2012, soit environ 0,1\% de l'épargne en France (Finansol, 2012). Si le volume reste faible, le taux de croissance interpelle: il souligne l'utilité de la finance solidaire dans la détection et le financement de projets économiques solvables, tout en prônant une dimension critique du fonctionnement du système financier et en proposant des innovations en termes de produits (comme les fonds communs de partage ou l'investissement social responsable), qui sont repris par la finance classique. Cette part reste pourtant marginale, du fait de la normalisation, de la banalisation ou de la disparition de formes de finance solidaire en fonction des contextes institutionnels.

Cependant, une analyse historique montre que la finance solidaire puise ses racines dans des expériences dès le début du XIX ${ }^{e}$ siècle en lien avec la construction du système financier. A cette époque, il s'agissait de financer des activités productives artisanales ou collectives. Aujourd'hui, l'enjeu est d'accompagner le renouvellement de l'entrepreneur individuel et de soutenir le développement des entreprises de l'économie sociale et solidaire (coopératives et associations principalement). Cette persistance atteste de sa réelle utilité dans le financement de l'économie en lien avec la diffusion des rapports marchands et capitalistes.

Dès lors, la finance solidaire n'est pas une finance éphémère, qui apparaît uniquement lors des crises du capitalisme. Dans certains cas, elle semble avoir un rôle palliatif dans l'accès au crédit professionnel et à l'entrepreneuriat, mais ces configurations ne peuvent faire oublier ses dimensions contestataires, voire transformatrices. Face aux caractéristiques du système financier conventionnel, elle est un mode de financement qui accompagne les évolutions des activités productives, en créant de nouvelles sources 
de financement et en innovant dans les procédures de leur attribution. La question centrale est donc de déterminer la nature des rapports entre la finance solidaire et le système financier, entre cloisonnement, complémentarité et intégration. Comme nous allons le démontrer par la suite, la finance solidaire est complémentaire au secteur financier en soutenant des projets économiques viables et en mobilisant une épargne éthique.

A partir d'une étude ciblée et représentative des projets et de la concrétisation d'initiatives financières solidaires sur une longue période - du début du $\mathrm{XIX}^{\mathrm{e}}$ siècle à nos jours - il s'agit d'interroger les raisons de leur existence, de leur durabilité et de leur évolution. Dans une première partie, l'analyse met en lumière la récurrence des contraintes de financement dans la construction du système financier. Une seconde partie étudie les formes historiques de la finance solidaire pour révéler les fonctions remplies. Enfin, la troisième partie distingue des caractéristiques de la finance solidaire et discute de son rôle dans l'évolution du système financier.

\section{La contrainte de financement}

L'existence d'inégalités dans l'accès aux services financiers professionnels est un fait régulier de notre système financier, aussi mature ou développé soit-il. La sélectivité du crédit et son corollaire le refus de celui-ci constituent en effet un phénomène inhérent aux comportements des banques sur le marché du crédit face à l'incertitude des relations financières et à l'organisation du système financier, créant ainsi une frange "d'emprunteurs insatisfaits» (Keynes, 1930, p. 212).

Une relation de crédit engage les individus dans une double livraison de monnaie dans le présent et le futur. Elle véhicule des représentations différentes de l'avenir: un risque concernant la probabilité de réalisation ou non d'un événement (le risque de non remboursement) ou une incertitude radicale rendant impossible de prédire l'ensemble des événements futurs (réalisation du chiffre d'affaires prévisionnel), comme Knight (1921) et Keynes (1921) le montrent. Ces représentations sont à l'origine d'une contrainte de financement: elle peut être un simple rationnement de crédit lorsque le risque est trop grand pour l'intermédiaire financier, comme le propose la théorie orthodoxe (Stiglitz, Weiss, 1981), ou une contrainte quand la banque et l'emprunteur ont peu de confiance dans l'avenir, comme le suggère la théo-

(2) Il existe des formes de crédit informel dans l'Europe précapitaliste (Fontaine, 2008) comme aujourd'hui dans les pays en développement (Duflo, 2010). rie hétérodoxe, post keynésienne en particulier (Wolfson, 1996). L'analyse historique de la construction du système financier en France confirme la présence continue d'une contrainte de financement pour des emprunteurs, celle-ci étant liée à la fois aux représentations et aux relations entre les banquiers et les emprunteurs et à l'organisation du système financier.

\section{Des emprunteurs solvables sans financement}

Au début du XIX siècle, l'utilisation du crédit bancaire professionnel se développe, mais il n'est pas accessible ${ }^{(2)}$ aux classes populaires et aux petits producteurs (artisans, commerçants, paysans): ne disposant pas d'épargne et des garanties nécessaires, les artisans, les ouvriers et les petits 
producteurs agricoles n'accèdent pas aux services bancaires. Le développement du système financier leur offre la possibilité de gérer leur épargne (par exemple les Caisses d'épargne) sans leur concéder du crédit (SaintMarc, 1983).

Son émergence répond principalement aux besoins de premiers industriels. Les services financiers sont réservés à une faible proportion de la population française, dans la distribution des crédits comme dans la collecte de l'épargne. Les activités de crédit sont principalement mises en œuvre par l'escompte, sur des courtes durées et avec des taux d'intérêt personnalisés. Au cours des premières phases de l'industrialisation, les crédits sont avant tout à court terme, sur des principes de renouvellement des facilités de trésorerie (Straus, 2011). Progressivement, les opérations dites capitalistiques via l'émission de titres participent au développement du financement direct. Cette offre est surtout destinée aux notables, aux grands capitalistes ou à l'Etat. Par exemple, la Banque de France n'émet aucune coupure inférieure à 500 francs avant les années 1840 et les montants inférieurs à 50 francs apparaissent après 1870 (Leclercq, 2010).

De façon structurelle, la relation financière se transforme, passant d'un crédit à forte dimension "interpersonnelle», fondé sur l'interconnaissance, à un crédit à dimension «impersonnelle» (Hautcœur, 2011). Dans le premier cas, la clientèle appartient à un cercle fermé composé de la famille, d'amis ou de relations d'affaires, tandis que, dans le second cas, la standardisation et l'industrialisation des services de crédit excluent les agents économiques ne répondant pas aux normes de la sélection marchande. La contrainte de financement se fonde sur les difficultés d'évaluation de la qualité des emprunteurs, cette dernière motivant la décision du prêteur en fonction du risque et de l'incertitude. Or le développement du système financier ne répond pas structurellement à ce phénomène.

La pauvreté monétaire ou les dotations initiales ne sont pas les seuls critères déterminants qui expliquent les difficultés de financement. Comme le montre Minsky (1957), les banques craignent de s'exposer à un risque d'insolvabilité et d'illiquidité ${ }^{(3)}$. Il s'agit plutôt d'un manque de confiance et de connaissances pour construire une évaluation du risque. Les catégories précitées sont rationnées, car elles présentent des risques élevés pour les intermédiaires financiers, qui méconnaissent ces formes organisationnelles. En d'autres termes, les entreprises en création, les toutes petites entreprises ou les organisations de l'économie sociale et solidaire ne suivent pas les règles de gouvernance, de propriété du capital et de redistribution de l'entreprise par capitaux. Elles présentent des spécificités

(3) Un agent est dit insolvable quand son patrimoine financier et ses actifs sont inférieurs à ses dettes. Il est en situation d'illiquidité, car il ne peut honorer ses créances à un moment donné cas ses actifs sont immobilisés (une maison, par exemple). mal perçues par le prêteur, alors qu'elles constituent des acteurs économiques à part entière (en termes de production, d'emploi, etc.). Ces difficultés au niveau de la construction de la relation entre les banquiers et les emprunteurs sont historiquement renforcées par l'organisation du système et sa régulation. Aujourd'hui encore, de nombreux entrepreneurs évoquent des difficultés d'accès au financement bancaire, en particulier les créateurs de petite entreprise (Guérin, 2002, p. 20). 


\section{Un système financier balkanisé et normatif}

Le système financier oscille entre un agencement institutionnel hiérarchique et balkanisé autour de cloisonnements sociaux, sectoriels et spatiaux et un agencement administratif et normatif des pratiques financières qui favorise des contraintes de financement. Au cours du XIX siècle, il s'organise et se structure, avec la création de nombreuses institutions bancaires et financières: notaires, banquiers d'affaires, Banque de France, banques commerciales, sociétés financières, bourse des valeurs (Lescure, Plessis, 1999). Cette diversité des intermédiaires favorise une organisation décentralisée et libéralisée du secteur.

Durant le $\mathrm{XX}^{\mathrm{e}}$ siècle, le développement de ce dernier s'opère sous la coupe des pouvoirs publics, qui encouragent une organisation centralisée et une concentration des intermédiaires financiers, entraînant une normalisation des critères de sélection des financements. Pourtant, le secteur reste caractérisé par une segmentation des marchés et des produits limités (Petit, Vernières, 1990). L'intervention de l'Etat, à différentes époques, ne répond pas à l'enjeu de l'accessibilité: il privilégie une relation instrumentale avec le système financier, qui se traduit par un contrôle des canaux du crédit, soit pour ses propres besoins (par exemple le financement de guerre ou de grands travaux au xixe siècle), soit pour favoriser le développement industriel. Dans les deux configurations, l'ensemble des besoins n'est pas couvert. La faiblesse des proximités géographique, organisationnelle et sociale réduit les échanges d'information et les liens de confiance entre les intermédiaires financiers traditionnels et une partie des agents économiques, soulevant des contraintes de financement.

Globalement, les évolutions dans la régulation du système financier, oscillant entre des périodes de libéralisation financière à la fin du XIX ${ }^{\mathrm{e}}$ siècle (Flandreau, Zumer, 2004) ou dans les années 80 et des périodes de régulation administrée du crédit (Aglietta, 2008), ont structurellement enraciné des phénomènes d'inadaptation de l'offre de financement par rapport aux besoins de certains agents économiques. Face à ces difficultés, la finance solidaire répond à la fois aux besoins d'entrepreneurs locaux individuels détenteurs de leurs moyens de production et aux entreprises collectives.

\section{Réponses non conventionnelles aux contraintes de financement}

La finance solidaire émerge et se structure face aux différentes manifestions de l'exclusion financière, qui se renouvelle selon les mutations institutionnelles du système financier. L'analyse historique que nous développons met en exergue des éléments de continuité et de discontinuité du XIX ${ }^{\mathrm{e}}$ siècle à nos jours, en France. Nous identifions en effet plusieurs initiatives alternatives qui s'apparentent à la finance solidaire depuis le début du XIX ${ }^{e}$ siècle, en raison de leur dimension "critique », parfois contestataire, par rapport au système financier conventionnel et de leurs questionnements quant à l'accessibilité sociale et au coût du financement. L'analyse des formes de finance solidaire depuis les années 80 s'inscrit dans la continuité de ces questionnements, tout en prenant des formes différentes. 


\section{De la contestation à l'affaiblissement}

Bien que les qualificatifs soient hétérogènes au XIX ${ }^{\mathrm{e}}$ siècle, plusieurs pratiques financières alternatives et solidaires interrogent le rôle des intermédiaires, en raison à la fois de leur pouvoir vis-à-vis des emprunteurs et du coût de cette mise en relation. Dès lors, la finance solidaire a mis en place des circuits courts entre les prêteurs et les emprunteurs afin de promouvoir des rapports de réciprocité dans les relations financières.

Au XIX ${ }^{e}$ siècle, l'expérience de la «banque d'échange » de Proudhon illustre le problème de la cherté du crédit (Ferraton, Vallat, 2011). Cette initiative a pour objectif de favoriser l'échange entre les producteurs de façon directe et réciprocitaire: elle fournit du crédit d'exploitation à court terme à des artisans et à des entrepreneurs individuels, en gageant leurs marchandises et leurs promesses de vente, introduisant ainsi la gratuité du crédit (Vallat, 1999). Plusieurs critiques ont mis en lumière des difficultés aussi bien dans l'émission des bons que dans leur circulation (Blanc, 2000), mais le fait que la banque d'échange ne fut jamais expérimentée laisse ces questions en suspens.

Face à la faiblesse ou à l'absence d'épargne de la classe ouvrière, la mobilisation des fonds étatiques semble indispensable pour certains défenseurs $d u$ mouvement coopératif. En 1831, Buchez sollicite, sans succès, l'Etat pour la création d'une caisse générale du crédit public afin d'offrir un crédit gratuit (Demoustier, Rousselière, 2005). Pour lui, l'Etat doit aider à la création d'associations ouvrières de production à l'initiative d'ouvriers qualifiés par la mise en place d'avances sans intérêt remboursables grâce aux réserves impartageables de l'association, issues des bénéfices dégagés par l'activité. L'objectif est la prise en charge du coût du crédit par l'Etat en proposant un intérêt nul dans des activités choisies.

De même, Louis Blanc, dans L'organisation du travail (1839), plaide pour un crédit d'Etat à taux zéro afin de financer les achats de matériel et d'assurer l'emploi et la production dans les ateliers sociaux. Il critique la position dominante du prêteur qui impose son intérêt à l'emprunteur, ce prix étant plus élevé quand le besoin financier est inéluctable (Blanc, 1847, p. 343-344). Face à cette situation, il suggère une intervention étatique qui se traduit par une nationalisation d'une banque et la mise en place d'un crédit gratuit pour les associations ouvrières. Pourtant, ces propositions restent sans suite. Parallèlement, le mouvement associationniste réfléchit sur l'autoorganisation des associations ouvrières de production, de leurs membres et de leurs sympathisants. Il s'agit de trouver des financements à plus long terme qui répondent aux besoins spécifiques des activités économiques sous forme collective.

\section{LA SOCIÉTÉ DU CRÉDIT AU TRAVAIL}

En 1863, Béluze fonde la Société du crédit au travail autour de trois fonctions : favoriser l'épargne des ouvriers, développer du crédit entre les membres et financer par le crédit et l'escompte les associations ouvrières de production (Vallat, 1999). Le sociétaire peut ainsi accéder à un crédit individuel au moins égal à sa quote-part dans la société. Il peut augmenter ce montant en constituant un groupe solidaire pour lequel le crédit collectif est supérieur 
à la somme des crédits individuels, car la solidarité du groupe augmente la solvabilité individuelle (Béluze, $1863 b$, p. 7). La société prend un intérêt proche du taux de l'escompte de la Banque de France et exerce un droit de contrôle sur les activités (participation au conseil d'administration). Toutes les opérations financières sont possibles, à l'exception des opérations de bourse et de spéculation (Béluze, 1863a, p. 71). La société fonctionne selon le principe «Une personne, une voix ». Une partie de ses bénéfices est redistribuée aux membres, tandis que $20 \%$ sont dédiés à un fonds de réserve sociale. Elle est créée le 27 septembre 1863, et compte trois ans plus tard 1100 associés. D’autres organisations associatives de financement se créentet reproduisent le modèle de Béluze selon une logique de proximité (par exemple la Stéphanoise, la Société lyonnaise de crédit au travail). Malgré une tentative de renflouement, la Société de crédit au travail fait faillite en novembre 1868, car elle ne peut plus honorer ses engagements, entraînant dans son sillage de nombreuses organisations coopératives. Cette défaillance est expliquée par le manque de ressources disponibles face à la demande de crédit et par les immobilisations à long terme nécessaires pour financer le développement des coopératives, alors que la banque dispose principalement de ressources à court terme. Des difficultés accentuées par le manque de compétences de ses gestionnaires (Gueslin, 1998).

\section{FAVORISER LA MUTUALISATION DE L'ÉPARGNE OUVRIÈRE}

A cette époque, l'idée d'une organisation collective qui favorise la mutualisation de l'épargne ouvrière se diffuse. Walras (1865) défend aussi les associations ouvrières de crédit à destination des travailleurs, car cette conception répond à des objectifs à la fois de justice sociale et d'intérêt individuel. Celles-ci facilitent la création d'un capital indivisible par le regroupement de cotisations volontaires et régulières des membres. D’une part, ces cotisations permettent de constituer un capital social et, d'autre part, les membres sont solidaires et offrent une garantie collective facilitant l'accès à des capitaux externes à la caisse. Ainsi, dans le cas d'un crédit personnel sans garantie réelle du travailleur, l'association assume le risque en offrant la garantie collective des membres et en engageant le capital social.

Par la suite, on assiste à la création de plusieurs réseaux de coopératives d'épargne et de crédit : en 1878, la Banque populaire d'Angers se constitue; en 1882, les caisses du Crédit mutuel d'Alsace-Lorraine sont créées; en 1885 naît la Société de crédit agricole de l'arrondissement de Poligny (Jura) [Gueslin, 1998]. Les membres sont des catégories professionnelles qui subissent des difficultés d'accès au crédit: les agriculteurs, les petits producteurs et les artisans. Ces coopératives se spécialisent en termes de philosophie (chrétienne, républicaine), de catégories socioprofessionnelles (paysans ou artisans) et d'implantation (rurale ou urbaine). Elles activent la proximité sous forme géographique (une région limitée, telle une commune ou une paroisse), relationnelle (l'encastrement des relations financières dans des réseaux sociaux), organisationnelle (le regroupement professionnel) et institutionnelle (le partage de valeurs). Grâce à plusieurs évolutions législatives, elles se structurent à un niveau régional, puis 
se regroupent pour articuler les circuits publics de financement à leurs circuits spécifiques coopératifs, ces évolutions ouvrant la voie vers des mutations plus profondes (Vienney, 1980).

Les coopératives d'épargne et de crédit véhiculent encore une relation de financement solidaire à la fin de la Seconde Guerre mondiale. Pourtant, le développement et l'élargissement des bénéficiaires, d'une part, et les transformations sectorielles du secteur bancaire, d'autre part, modifient structurellement l'organisation des relations de financement. Ces transformations détruisent la relation de financement solidaire des coopératives d'épargne et de crédit sans laisser d'opportunité pour la création de modèles autonomes de finance solidaire. Aujourd'hui, les banques coopératives n'appartiennent plus à cette dernière, mais elles contribuent à son émergence et à son développement (Richez-Battesti, Gianfaldoni, 2006).

\section{Entre continuités et discontinuités}

Comme dans le passé, la finance solidaire contemporaine repose sur trois principales critiques par rapport au système financier: la perte de contrôle des épargnants, le manque de financement pour des projets économiques alternatifs et l'exclusion de certains agents économiques. D’abord, la déstabilisation du modèle de croissance des Trente Glorieuses ravive les critiques vis-à-vis du système financier affaiblissant de plus en plus le contrôle des épargnants. Des clubs d'investisseurs pour une gestion alternative locale de l'épargne solidaire (Cigales) émergent, dans la continuité des formes de contestation du siècle précédent. Ils souhaitent se réapproprier le contrôle de l'utilisation de leur épargne et dédier celle-ci à des projets économiques alternatifs (commerce équitable, agriculture biologique, éducation, culture) [Taupin, Glémain, 2007]. Les clubs sont ancrés dans la proximité par la mise en place d'un circuit court entre les épargnants et l'emprunteur, et ils renouent avec la logique de la réciprocité et de solidarité privée plus affinitaire qui favorise la gratuité du financement.

La finance solidaire contemporaine prend aussi racine dans le mouvement coopératif, avec plusieurs sociétés coopératives financières solidaires qui rapprochent des épargnants solidaires désirant financer des entreprises d'utilité sociale au niveau local. Celles-ci ont un rôle d'accompagnement, d'expertise et de gestion financière solidaire. Elles prennent racine soit dans la contestation du fonctionnement du système financier (la Nef ou Garrigue, par exemple), soit dans la volonté de soutenir le développement local (Autonomie et Solidarité, IES). La dimension territoriale est très marquée dans plusieurs cas (Artis, 2007).

Enfin, face à l'exclusion financière des individus, des associations (Plateforme Initiative Locale, en 1985; Adie, en 1989) développent le microcrédit afin de favoriser l'accès à des prêts de faible montant pour des emprunteurs sans épargne préalable et sans garantie. Celles-ci se focalisent sur le financement de la création d'activité sous toutes formes d'entreprises. Ces associations de microfinance soutiennent la petite production marchande par l'aide à la formalisation d'activités économiques nécessitant peu de capital de départ (Vallat, 1999). 


\section{Circuits courts, gouvernance démocratique ET HYBRIDATION DES RESSOURCES}

La comparaison historique montre que la mise en place de circuits courts de financement solidaire constitue un élément fondateur, qui a plusieurs effets face à la contrainte de financement. D'abord, ils sont moins coûteux que les autres circuits de financement, et les processus de financement s'appuient sur des critères de sélection des projets et d'évaluation qui se distinguent des critères marchands, privilégiant la confiance sociale et l'expression des besoins.

La finance solidaire repose sur la participation des épargnants privés dans la gouvernance des organisations: présence aux comités d'engagement des crédits, accompagnement des emprunteurs, représentations publiques de la structure, etc. Cette participation permet de renouveler l'implication et le contrôle des épargnants dans les décisions financières. C'est aussi un moyen de réactiver les liens sociaux de proximité, favorisant la confiance et le partage d'informations. La mutualisation renforce la solidarité entre les emprunteurs et les apporteurs de fonds.

Ensuite, ces initiatives reposent sur la combinaison de ressources marchandes, non marchandes et non monétaires, grâce aux alliances avec les acteurs publics et privés (épargnants individuels, banques, entreprises), ces partenaires participant financièrement à la prise en charge du coût total du financement (coût financier et d'accompagnement). Ces initiatives constituent une mise de fonds importante en l'absence d'épargne préalable pour les emprunteurs: elles mutualisent des fonds pour créer des garanties solidaires partenariales et diviser ainsi la prise de risque des prêteurs.

Ainsi, face aux problèmes d'inaccessibilité aux services financiers, la finance solidaire crée une offre de financement privé fondée sur des garanties collectives qui mobilise une épargne faiblement "intermédiée » et peu coûteuse. Ce modèle est tout à fait pertinent pour soutenir une filière ou un secteur d'activité, car il permet de développer des outils financiers adaptés aux spécificités de la filière, mais aussi d'améliorer l'expertise des gestionnaires des fonds. Cependant, cette forte similitude est aussi source de fragilité en cas de déstabilisation de la filière. Les projets économiques individuels sont plus nombreux que les projets collectifs et alternatifs, affaiblissant l'effet levier pour un changement de modèle que propose la finance solidaire.

\section{La finance solidaire: en complémentarité avec le système financier}

La pérennité de la finance solidaire invite à s'interroger sur son rapport avec le système financier et économique et à mettre en lumière ses apports. Celle-ci s'appuie sur des structures de proximité qui constituent un capital collectif et offrent des services financiers socialisés. Dès lors, elle est complémentaire au système financier : elle construit de nouvelles réponses à destination des agents productifs exclus du fait des transformations de la régulation financière, prenant ainsi en charge des espaces de financement périphériques nécessaires à la durabilité 
du système financier dominant. Cette complémentarité ne se limite pas à pallier les dysfonctionnements du système financier, elle vise à explorer des formes alternatives qui participent à la régulation de ce dernier.

\section{Favoriser l'inclusion financière}

Les conditions de financement de la finance solidaire favorisent l'inclusion financière. Pour les emprunteurs, les conditions monétaires et non monétaires sont annoncées et fixées avant la décision de financement; elles ne résultent pas d'une négociation dans un rapport de force inégal entre l'emprunteur et l'intermédiaire financier, comme souvent dans le cas d'un financement bancaire. Le taux d'intérêt comme les garanties sont en effet identiques pour les emprunteurs dans la microfinance, par exemple. Les taux d'intérêt sont aussi très stables dans les coopératives financières, comme la Nef ou Garrigue. Les financements solidaires sont moins discriminants pour l'emprunteur, car ils offrent un taux d'intérêt identique pour tous, moins sensible au niveau de risque individuel.

Concernant la collecte de fonds, les ressources proviennent des parts sociales, pour les coopératives financières; de la mutualisation de l'épargne, pour les circuits courts; de la mobilisation de l'épargne salariale ou de fonds abondés par les banques, pour les associations de microfinance. Ces contributions s'inscrivent dans une perspective de long terme. Elles sont peu fluctuantes et demandent des rémunérations raisonnables, permettant ainsi de créer des financements longs et moins coûteux pour les entreprises.

De ce fait, la finance solidaire permet de réduire le coût: soit par la gratuité, dans les circuits courts; soit par la mutualisation et la collecte d'une épargne privée militante, dans les sociétés financières coopératives; soit par l'alliance entre des acteurs privés et publics, dans les coopératives financières. Parallèlement au taux d'intérêt, la finance solidaire se démarque en substituant les garanties matérielles par plusieurs formes de garantie solidaire. Il en existe trois, qui coexistent: la cotisation à un fonds de garantie solidaire, la souscription à des organismes de garantie solidaire et la mise en place d'une caution solidaire. Paradoxalement, ces conditions sont peu personnalisées: le taux d'intérêt, la durée ou la garantie sont identiques pour tous les emprunteurs, réduisant l'inégalité de la relation de négociation et de financement. Ainsi, ces conditions contractuelles tendent à être moins sensibles aux conditions et aux évolutions conjoncturelles du marché du financement. En d'autres termes, la finance solidaire est moins volatile et spéculative que les autres intermédiaires financiers. Elle a pour objectif d'intégrer l'emprunteur dans une logique financière sans dégrader sa future capacité de financement.

\section{La place des partenariats dans la différenciation}

La mobilisation de parties prenantes, au-delà du cercle affinitaire, est fondamentale aux dépassements de la contrainte de financement. Comme nous l'avons montré, les premiers banquiers s'adressent aux populations aisées, du même milieu sociologique, reproduisant les cloisonnements sociaux. La finance solidaire s'appuie sur des relations d'appartenance et de filiation proches des pratiques financières informelles (tontines, prêts 
communautaires), puis se développe grâce à des produits financiers standardisés, tout en maintenant des relations interpersonnelles dans l'accompagnement entre les bénévoles et les emprunteurs et dans le cautionnement par la mobilisation de l'entourage de l'emprunteur. Les partenariats avec les banques et les collectivités territoriales ont favorisé la normalisation des pratiques financières et leurs spécifications en fonction des projets (individuels ou collectifs, locaux ou innovants) ou des financements (prêt ou investissement). Ils témoignent de l'imbrication de la finance solidaire dans le système financier, tout en interrogeant les rapports de force, entre concurrence, instrumentalisation ou complémentarité. Ils permettent aussi de comprendre les phénomènes de diffusion de certaines caractéristiques de la finance solidaire (Artis, 2012), qui expliquent l'illusion d'une intégration dans le système financier classique. Aujourd'hui, les produits d'épargne solidaire, la microfinance et les autres exemples de circuits courts de financement solidaire émanent d'établissements financiers conventionnels, de groupes coopératifs, de sociétés financières lucratives ou d'associations. Ces produits sont des éléments de différenciation des intermédiaires financiers afin de capter une population d'emprunteurs rentables et de séduire des épargnants plus volatiles et difficiles à fidéliser. Pourtant, les conditions de création des produits, les règles internes de gestion de ces fonds et celles de redistribution distinguent encore fortement la finance solidaire des intermédiaires financiers classiques.

\section{Territorialisation et décentralisation encouragent la stabilité}

La finance solidaire repose sur la décentralisation et la territorialisation des relations de financement entre les individus et les organisations. D'une part, des structures nationales favorisent la création d'associations territoriales, de délégations locales et de permanences. D’autre part, des structures territoriales de capital risque solidaire existent. De ce fait, la prise de décision du financement et la mise en place du lien social sont gérées localement à travers la mobilisation des réseaux de proximité. Cette caractéristique la différencie des autres intermédiaires financiers qui, de part l'évolution du système financier, ont privilégié une déterritorialisation de leurs activités. Aujourd'hui, le rapport au territoire et à son renouvellement est un enjeu du débat sur la régulation du système financier (Lordon, 2009).

A travers ces relations de proximité, la finance solidaire participe à la définition et à la prise en compte des besoins locaux et sociaux, à l'articulation de l'hétérogénéité du territoire (cohésion sociale) et à l'organisation d'activités et de production de ressources spécifiques. Néanmoins, il ne faut pas amoindrir l'existence de limites dans la territorialisation de la finance solidaire (faible mobilisation de l'épargne directe, difficultés de pérennité des structures): cette dernière irrigue peu les territoires les plus en déclin ou les zones en difficulté, se développant plutôt sur les territoires déjà dynamiques (Glémain et al., 2010).

La finance solidaire contribue à sécuriser l'offre de financement en associant les relations financières à des relations sociales et en activant des proximités en faveur d’investissements productifs locaux. Par ces mécanismes, 
elle permet une meilleure affectation des ressources et favorise les comportements financiers coopératifs. Elle assure un accès au financement envers ceux qui, à cause de leur situation financière et de leur condition sociale, sont exclus du système bancaire par la combinaison de principes marchands et non marchands et la construction d'un compromis sociofinancier.

Ces éléments favorisent une certaine stabilité dans l'offre de financement de proximité et permettent de maintenir un contrôle de l'utilisation des financements solidaires. Dès lors, elle soutient des activités financières en accompagnant les mutations structurelles et en socialisant les risques et les coûts du financement d'activités économiques de proximité.

\section{Conclusion}

La finance solidaire est un phénomène souvent réduit à un financement éphémère. Pourtant, les observateurs, politiques comme économistes, semblent ne pas mesurer ses apports dans la construction du système financier et dans la vivacité du système économique, en particulier pour certaines formes organisationnelles. La finance solidaire est souvent invisible, alors qu'elle est un système de financement pérenne et actif en période de crise comme de croissance. Aujourd'hui, le renouveau de la finance solidaire prend racine dans un accroissement de la sélection bancaire et une normalisation des relations bancaires selon une régulation concurrentielle. Le contexte institutionnel actuel amoindrit les espaces de socialisation au profit d'un processus de normalisation et d'institutionnalisation qui favorise des formes de garantie et d'assurance individuelles plutôt que collectives.

Le système financier et bancaire, régi par des principes exclusivement marchands et capitalistes, tend en effet à exclure des emprunteurs entrepreneurs. La finance solidaire, par la mise en place de structures financières associatives et coopératives, propose de faciliter la régulation du crédit en mutualisant l'épargne, en sécurisant les risques entre les prêteurs et en combinant des principes de gestion marchands et non marchands. Létude de ces pratiques conclut à repenser la dimension sociale du crédit et sa fonction dynamique au cœur des activités économiques et à relocaliser les activités financières pour le soutien d'activités productives locales en vue d'améliorer le bien-être collectif et individuel. De plus, la finance solidaire invite à davantage de coopération entre les prêteurs (banques, Etat, épargnants individuels) afin de mutualiser les ressources et les risques, car l'accès au crédit contribue à l'amélioration du bien-être collectif.

Mais il ne s'agit pas d'attribuer à la finance solidaire des challenges qu'elle ne peut assumer. Elle n'apporte pas de réponse globale dans notre période de crise. Elle peut, d'une part, amortir les effets de la crise sur les publics les plus fragiles en assurant un accès au crédit et, d'autre part, développer ces pratiques solidaires pour les diffuser plus largement. 


\section{BibLIOGRAPHIE}

Aglietta M., 2008, La crise: pourquoi en est-on arrivé là ? Comment en sortir? Paris, Michalon. Artis A., 2012, La finance solidaire: analyse socio-économique d'un système de financement, éditions Houdiard, Paris.

Artis A., 2007, « La « finance solidaire territorialisée »: une réponse de proximité en faveur de la construction d'un territoire de référence dans un processus de mondialisation ", Cahier du Ceren, ${ }^{\circ} 19$, p. 20-40.

Blanc J., 2000, Les monnaies parallèles: unité et diversité du fait monétaire, L’Harmattan, Paris Blanc L., 1847, L'organisation du travail, Société de l'industrie fraternelle, Paris.

Béluze J.-P., 1863a, Les associations, conséquences du progrès social, Crédit au travail, janvier, Paris.

Béluze J.-P., 1863b, Qu'est ce que la Société du Crédit au travail?, librairie Guillaumin et Cie, Paris.

Demoustier D., Rousselière D., 2005, « L'économie sociale et coopérative, des associationnistes: de la critique des fondements de l'économie politique à la régulation sociale du marché », Annals of public and cooperative economics, vol.76, issue 4, p. 517-543, décembre. Duflo E., 2010, La politique de l'autonomie: lutter contre la pauvreté (II), Paris, Le Seuil, République des idées.

Ferraton C., Vallat D., 2011, « Une approche politique du crédit populaire: Pierre-Joseph Proudhon et le crédit mutuel ", Cahiers d'économie politique, $\mathrm{n}^{\circ} 60$, p. 45-65.

Finansol, 2012, Baromètre de la finance solidaire, Finansol-La Croix, Paris.

Flandreau M., Zumer F., 2004, Les origines de la mondialisation financière, 1880-1913, OECD Publishing, Paris.

Fontaine L., 2008, L'économie morale: pauvreté, crédit et confiance dans l'Europe préindustrielle, Gallimard, coll. « Essai », Paris.

Glémain P., Bioteau E., Artis A., 2010, Finances solidaires et territoires : analyses en Bretagne et en pays de la Loire, Revue économique rurale et urbaine, mai, p. 213-233.
Guérin I., 2002, « La microfinance et la création d'entreprise par les chômeurs: la situation dans quelques pays européens et en Amérique du Nord ", rapport pour le Bureau international du travail et le secrétariat d'Etat à l'économie solidaire, mars, $107 \mathrm{p}$.

Gueslin A., 1998, L'invention de l'économie sociale: idées, pratiques et imaginaires coopé-

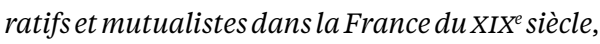
deuxième édition révisée et augmentée, Paris, Economica.

Hautcœur P.-C., 2011, « Les transformations du crédit en France au XIX ${ }^{\mathrm{e}}$ siècle », Romantismes, 151, 1, p. 23-38.

KeynesJ. M.,1930, A Treatise on money, London, in The Collected writings of John Maynard Keynes, vol. VI, The applied theory of money, Cambridge university press, 1971, New York.

Keynes J. M., 1921, Treatise on probability, Collected writings, vol. VIII, Cambridge university press, Cambridge.

Knight F., 1921 [1965], Risk, uncertainty and profit, New York, Harper Torchbooks.

Leclercq Y., 2010, La banque supérieure: la Banque de France de 1800 à 1914, Garnier, Paris.

Lescure M., Plessis A. (dir), 1999, Banques locales et banques régionales en France au $X I X^{e}$ siècle, Albin Michel, Paris.

Lordon F., 2009, "Après la crise financière: "réguler" ou refondre? ", Revue de la régulation, $\mathrm{n}^{\circ} 5,1^{\mathrm{er}}$ semestre, Spring.

Minsky H. P., 1957, " Monetary systems and accelerator models ", The American economic review, vol. XLVII, n 6, p. 859-883.

Petit P., Vernières M., 1990, « La banque et ses emplois: un service en transition ", Travail et Emploi, $\mathrm{n}^{\circ}$ 44, p. 7-18.

Richez-Battesti N., Gianfaldoni P. (dir.), 2006, Les banques coopératives en France: le défi de la performance et de la solidarité, L'Harmattan, Paris.

Saint-Marc M., 1983, Histoire monétaire de la France, 1800-1980, presses universitaires de France, Paris. 
Stiglitz J. E., Weiss A., 1981, « Credit rationing in markets with imperfect information ", American economic review, vol. 71 (June), p. 393-410. Straus A., 2011, "Banques et industries en France et en Angleterre: des années 1880 à la Seconde Guerre mondiale ", Revue d'économie financière, $\mathrm{n}^{\circ}$ 104, p. 35-50.

Taupin M.-T., Glémain P., 2007, « Les logiques d'acteurs des finances solidaires contemporaines: entre innovation et résilience ", Annals of public and cooperative economics, 78 (4), p. 629-661.
Vallat D., 1999, «Exclusion et liens financiers de proximité, financement de micro-activités ", thèse de doctorat, université Lyon II, décembre, $525 \mathrm{p}$.

Vienney C., 1980, Socio-économie des organisations coopératives, deux tomes, Ciem, Paris.

Walras L., 1865, Les associations populaires de consommation, de production et de crédit, éditions Dentu, Paris.

Wolfson M. H., 1996, « A post keynesain theory of credit rationing », Journal of post keynesian economics, printemps, vol. $18, \mathrm{n}^{\circ} 3$, p. 443-470. 